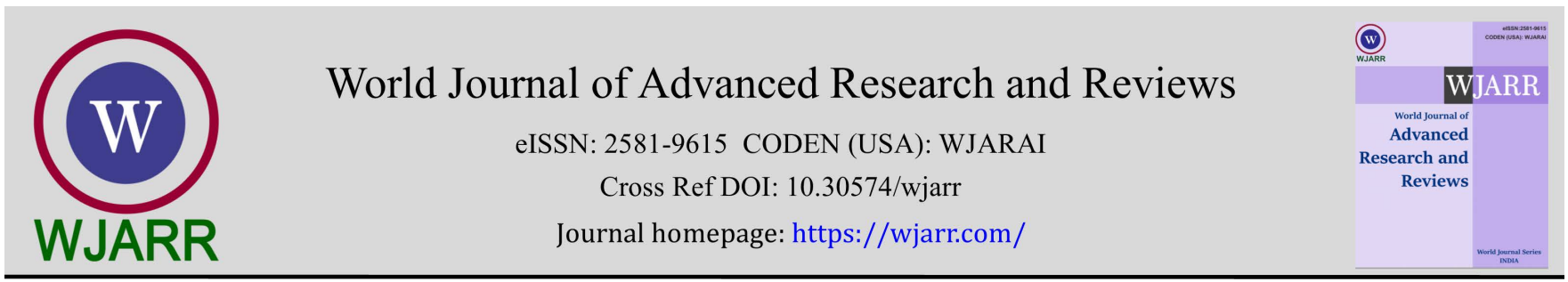

(CASE STUdy)

Check for updates

\title{
First case of Streptococcus pluranimalium meningitis isolated at Kibogora district hospital
}

\author{
Jean Claude Uwimana * and Innocent Nzabahimana \\ ${ }^{1}$ Kibogora District Hospital, Rwanda. \\ 2 National Reference Laboratory, Rwanda. \\ World Journal of Advanced Research and Reviews, 2021, 09(02), 169-171
}

Publication history: Received on 13 January 2021; revised on 09 February 2021; accepted on 11 February 2021

Article DOI: https://doi.org/10.30574/wjarr.2021.9.2.0050

\begin{abstract}
Streptococcus pluranimalium has mainly been found to cause infections in animals and was rarely isolated in humans. Very few cases of streptococcus pluranimalium have been reported in the literature. We report a first case of streptococcus pluranimalium meningitis in a pediatric patient. This patient was treated successfully with a combination of $3^{\text {rd }}$ generation cephalosporin and chloramphenicol since her first day of admission. Only 4 of the reported human infections caused by Streptococcus pluranimalium were pediatric cases. We didn't find neither adult nor pediatric cases of meningitis caused by this new novel species of Streptococcus pluranimalium. Clinical doctors have to remember that a rare pathogen like Streptococcus pluranimalium isolated primarily in animals can be a causative agent of human infection. More diagnostic methods are needed to detect early what could be rare causative germs of human infection. And when streptococcus pluranimalium is isolated, the first line antibiotics will be vancomycin, carbapenems, linezolid, aminoglycosides, and $3^{\text {rd }}$ generation cephalosporins.
\end{abstract}

Keywords: Streptococcus pluranimalium; Meningitis; Pediatric; Treatment

\section{Introduction}

Streptococcus pluranimalium is unusual streptococcal species, which is rarely isolated from human infection [1]. It was first described by Devriese et al. in 1999 isolated from genital tract and tonsils of cattle, tonsils of a goat and a cat and the excreta and respiratory tract of canaries. It primarily causes infections in bovine and avian species $[1,2,3,4,6$, 8].Clinicians should be aware of an increasing incidence of non-hemolytic Streptococcal infections. [1] We report the first case of Streptococcus pluranimalium meningitis isolated at Kibogora district hospital.

\section{Case presentation}

A 4 year old female patient previously healthy presented at Kibogora district hospital on $29^{\text {th }}$ July 2020 with abdominal pain, fever, vomiting, agitations and seizures. On physical exam, Heart Rate $(\mathrm{HR})=123 \mathrm{bpm}$, Temperature $(\mathrm{T})=38.4^{\circ}$ C, Sp02 $=97 \%$, weight $=16 \mathrm{~kg}$. She had neck stiffness and positive Kerning and Brudzinski signs. The Blantyre score was $4 / 5$.

The differential diagnosis were Meningitis and Malaria.

We requested Full blood count, thick smear for malaria and lumbar puncture. While waiting for results of laboratory exams, the patient was treated with ceftriaxone then after 2 days with cefotaxime as there was stock out of ceftriaxone

\footnotetext{
${ }^{*}$ Corresponding author: Jean Claude Uwimana

Anesthesiologist, KIBOGORA District Hospital, Rwanda.
}

Copyright (C) 2021 Author(s) retain the copyright of this article. This article is published under the terms of the Creative Commons Attribution Liscense 4.0. 
and she was treated also with chloramphenicol, paracetamol and IV fluids. When results of laboratory exams came out, the thick smear for malaria was negative, full blood count showed leukocytosis with neutrophilia and there were Gram positive cocci on lumbar puncture. The decision was to continue with antibiotics the patient was getting and IV fluids while waiting for the results of culture of cerebrospinal fluid (CSF). The patient improved with no more leukocytosis nor neutrophilia but she had post meningitis deafness. She was discharged 14 days after her admission at KIBOGORA district hospital. And her caretaker was given a rendez - vous to bring back the patient after 2 weeks for follow up of post meningitis deafness.

Results of CSF culture which came out 16 days after patient admission at KIBOGORA district hospital showed Streptococcus pluranimalium sensitive to linezolid, cefotaxime, chloramphenicol and ciprofloxacin as shown in the table below:

\begin{tabular}{|c|c|c|}
\hline \multicolumn{2}{|c|}{ TEST REQUESTED } & \multirow{2}{*}{$\begin{array}{c}\text { RESULT } \\
\text { Streptococci SPP }\end{array}$} \\
\hline CSF Culture & serotyping & \\
\hline Gram Stain & Gram stain result & Gram Positive Cocci \\
\hline \multirow{9}{*}{ Sensitivity } & Linezolid & Sensitive \\
\hline & Gentamicin & Resistant \\
\hline & Meropenem & Sensitive \\
\hline & Imipenem & Sensitive \\
\hline & Streptomycin & Resistant \\
\hline & Chloramphenicol & Sensitive \\
\hline & Cefotaxime & Sensitive \\
\hline & Ciprofloxacin & Sensitive \\
\hline & AFB & No Acid Fast Bacilli \\
\hline
\end{tabular}

CSF Analysis results

The caretaker brought the patient 1 month after patient's discharge for follow up of post meningitis deafness and it was found that the patient had no more deafness.

\section{Discussion}

The Streptococcus pluranimalium is an uncommon streptococcus species, which has been isolated and reported from human and animal infections. The term of pluranimalium indicates that bacterium is able to cause infections in different animals [1,3]. Since its first description, this organism has been identified as a causal pathogen in farm animals [3].

Despite the reports about various infections in animals by $S$ pluranimalium, the reports about human infections are limited, and it is not clear that this microorganism is either the initial pathogen or not [1]. To the best of our knowledge, among the previously reported 13 human infections caused by Streptococcus pluranimalium, 3 were solely isolated from brain abscesses, 3 from septicemia, 2 from endocarditis [1, 7], 1 from pericoronitis [6], 1 from ventilator-associated pneumonia, 1 from submandibular abscess [7], 1 from peritoneal dialysis peritonitis [8] and the last one from both infective endocarditis and brain abscess [2]. Only 4 of the reported human infections caused by Streptococcus pluranimalium were pediatric cases. We didn't find neither adult nor pediatric cases of meningitis caused by this new novel species of Streptococcus pluranimalium. And there is a high probability that our patient represents the first case of Streptococcus pluranimalium as the new causative agent of meningitis.

Based on results of previous studies, it seems that Streptococcus pluranimalium is sensitive to vancomycin, carbapenems, linezolid, aminoglycosides, and $3^{\text {rd }}$ generation cephalosporins [1]. In addition to being sensitive to carbapenems and $3^{\text {rd }}$ generation cephalosporins, the species isolated from our national reference laboratory was sensitive to chloramphenicol and ciprofloxacin. Our patient was treated with combination of both 3rd generation 
cephalosporins and chloramphenicol since her first day of admission in our hospital and when results of cerebrospinal fluid analysis came out, her clinical status had improved and this shows that in a low resource setting when meningitis or another human infection is suspected in a patient whose clinical status is not improving using large spectrum antibiotics like $3^{\text {rd }}$ generation cephalosporins or carbapenems can help to prevent other potential complications including death that can occur from a poorly treated infection.

\section{Conclusion}

The present study has demonstrated that Streptococcus pluranimalium can be the new causative agent of meningitis. It aims to remind clinical doctors of the rare pathogen of Streptococcus pluranimalium as a causative agent of human infection. More diagnostic methods are needed to detect early what could be rare causative germs of human infection.

\section{Compliance with ethical standards}

\section{Acknowledgments}

We would like to thank Kibogora district hospital staff who were involved in the management of this patient we presented as case report. Special thanks to Rwanda National Reference Laboratory especially Innocent NZABAHIMANA who informed to Kibogora district hospital staff about the laboratory results.

\section{Disclosure of conflict of interest}

Authors declare no conflict of interest.

\section{Statement of informed consent}

Informed consent was obtained from all individual participants included in the study.

\section{References}

[1] Ghazvini K, Karbalaei M, Kianifar H, Keikha M. The first report of Streptococcus pluranimalium infection from Iran: A case report and literature review. Clin Case Rep. 2019; 7: 1858-1862.

[2] Parikshit Duriseti, Jorge Fleisher. Streptococcus pluranimalium infective endocarditis and brain abscess. IDCases. 2019; 18.

[3] Abeera Ahmed, Gohar Zaman, Adeel Gardezi, Luqman Satti, Nargis Sabir and Aliya Haleem. Cerebral abscess caused by novel species: Streptococcus pluranimalium. Journal of the College of Physicians and Surgeons Pakistan. 2018; 28.

[4] Richard Facklam. What Happened to the Streptococci: Overview of Taxonomic and Nomenclature Changes? Clin. Microbiol. Rev. 2002; 15.

[5] Fotoglidis A, Pagourelias E, Kyriakou P, Vassilikos V. Endocarditis caused by unusual Streptococcus species (Streptococcus pluranimalium ). HIPPOKRATIA. 2015; 19(2): 182-185.

[6] Mariia O. Faustova, Maiia M. Ananieva, Galina A. Loban', Oksana O. Dobrovolska, Yaroslav O. Basarab, Ruslan V. Petrenko. Streptococcus pluranimalium as a new causative agent of acute pericoronitis: a rare case report. Euromediterranean Biomedical Journal. 2019;14(14): 062-064.

[7] Mónica Mercado, Franco Oseguera, Pedro Martínez, Martín Guerrero, Cesar Juárez, José Santos. Streptococcus pluranimalium , primeros casos pediátricos en México. Revista Biomédica. 2019.

[8] Durga Arinandini Arimuthu, Christopher Thiam Seong Lim. Streptococcus plurianimalium Peritonitis in a Patient on Continuous Ambulatory Peritoneal Dialysis: A Case Report. Mal J Med Health Sci. 2020; 16(4): 388-390. 